Proyecciones

Vol. 25, No 3, pp. 293-306, December 2006.

Universidad Católica del Norte

Antofagasta - Chile

DOI: 10.4067/S0716-09172006000300006

\title{
CONVERGENCE OF NEWTON'S METHOD UNDER THE GAMMA CONDITION
}

\author{
IOANNIS K. ARGYROS \\ CAMERON UNIVERSITY, U.S.A.
}

Received : April 2006. Accepted : October 2006

\begin{abstract}
We provide a semilocal as well as a local convergence analysis of Newton's method using the gamma condition [1], [10], [11]. Using more precise majorizing sequences than before [4], [8]-[11] and under at least as weak hypotheses, we provide in the semilocal case: finer error bounds on the distances involved and an at least as precise information on the location of the solution; in the local case: a larger radius of convergence.
\end{abstract}

AMS (MOS) Subject Classification Codes : 65H10, 65G99, $47 H 17,49 M 15$.

Key Words: Banach space, Newton's method, local/semilocal convergence, Newton-Kantorovich theorem, Fréchet derivative, majorizing sequence, radius of convergence, gamma condition, analytic operator. 


\section{Introduction}

In this study we are concerned with the problem of approximating a locally unique solution $x^{*}$ of equation

$$
F(x)=0,
$$

where $F$ is a Fréchet-differentiable operator defined on a convex subset $D$ of a Banach space $X$ with values in a Banach space $Y$.

The most popular method for generating a sequence $\left\{x_{n}\right\}(n \geq 0)$ approximating $x^{*}$ is Newton's method given by

$$
x_{n+1}=x_{n}-F^{\prime}\left(x_{n}\right)^{-1} F\left(x_{n}\right) \quad(n \geq 0) \quad\left(x_{0} \in D\right),
$$

where $F^{\prime}(x) \in L(X, Y)$ the space of bounded linear operators from $X$ into $Y$. A survey on local as well as semilocal convergence results for Newton's method (1.2) can be found in [2], [3]-[10], and the references there.

In the International Congress of Mathematicians held in 1986, Smale [8] proposed to use the analytic property of operator $F$ to replace the domain condition in the Newton-Kantorovich theorem [3], [7], in order to determine the convergence of Newton's method by thoroughly making use of the information of $F$ at the initial point $x_{0} \in D$. This work is of great theoretical interest.

Assuming $F^{\prime}\left(x_{0}\right)^{-1} \in L(Y, X), F$ is analytic at $x_{0} \in D$,

$$
\alpha=\beta \gamma\left(F, x_{0}\right)
$$

where,

$$
\beta=\left\|F^{\prime}\left(x_{0}\right)^{-1} F\left(x_{0}\right)\right\|
$$

and

$$
\gamma\left(F, x_{0}\right)=\sup _{k \geq 2}\left\|F^{\prime}\left(x_{0}\right)^{-1} \frac{F^{(k)}\left(x_{0}\right)}{k !}\right\|^{\frac{1}{k-1}},
$$

then Smale's main result can be described as follows:

Theorem 1.1 (Smale [8]). Suppose $\frac{\alpha}{\left(2 \alpha^{2}-4 \alpha+1\right)^{2}}=q<1$, then Newton's method (1.2) starting at $x_{0}$ is well defined, and

$$
\left\|x_{n+1}-x_{n}\right\| \leq q^{2^{n}-1} \beta \quad(n \geq 0) .
$$

In light of this theorem, Smale pointed out that there exists a constant $\alpha_{0} \cong .130707$ such that

$$
\left\|x_{n+1}-x_{n}\right\| \leq\left(\frac{1}{2}\right)^{2^{n}-1} \beta \quad(n \geq 0) .
$$


In 1989, by introducing the majorizing sequence method into the point estimation, X. Wang and D. Han obtained the following semilocal convergence result which is more precise than Theorem 1.1.

Theorem 1.2 [10]. If

$$
\alpha \leq 3-2 \sqrt{2},
$$

then sequence $\left\{x_{n}\right\}(n \geq 0)$ generated by Newton's method (1.2) is well defined, remains in

$$
\left.\bar{U}\left(x_{0},\left(1-\frac{1}{\sqrt{2}}\right) \frac{1}{\gamma\left(F, x_{0}\right)}\right)\right)
$$

for all $n \geq 0$ and converges to a unique solution $x^{*}$ of equation $F(x)=0$ in $\bar{U}\left(x_{0}, s^{*}\right)$ so that

$$
\left\|x_{n+1}-x_{n}\right\| \leq s_{n+1}-s_{n}
$$

and

$$
\left\|x_{n}-x^{*}\right\| \leq s^{*}-s_{n},
$$

where,

$$
\begin{aligned}
s_{n+1} & =s_{n}-\frac{f\left(s_{n}\right)}{f^{\prime}\left(s_{n}\right)} \quad(n \geq 0), \quad s_{0}=0 \\
s^{*} & =\lim _{n \rightarrow \infty} s_{n}=\frac{1+\alpha-\sqrt{(1+\alpha)^{2}-8 \alpha}}{4 \gamma\left(F, x_{0}\right)}
\end{aligned}
$$

and

$$
f(t)=\beta-t+\frac{\gamma t^{2}}{1-\gamma\left(F, x_{0}\right) t}, \quad t<\frac{1}{\gamma\left(F, x_{0}\right)} .
$$

The constant $3-2 \sqrt{2}$ in this theorem is optimum under that condition, and the resulted conclusion is also the best one. Since $3-2 \sqrt{2} \cong .171573>$ .130707 , this result is evidently an improvement of that of Smale's.

Clearly, the above two results are based on the assumption that the sequence

$$
\left\|\frac{F^{\prime}\left(x_{0}\right)^{-1} F^{(n)}\left(x_{0}\right)}{n !}\right\|, \quad(n \geq 2)
$$

is bounded above by

$$
\sup _{k \geq 2}\left\|\frac{F^{\prime}\left(x_{0}\right)^{-1} F^{(n)}\left(x_{0}\right)}{n !}\right\|^{\frac{1}{n-1}} .
$$


However this kind of assumption may not be reasonable particularly, for some concrete and special operators appearing in connection with the DurandKerner method, it is really so [9].

An attempt has been made by X. Wang [11] to avoid such an assumption. In particular Wang proposed the gamma $\gamma$-condition:

Definition 1.3. Suppose $\gamma>0$. We say $F$ satisfies the $\gamma$-condition at $x_{0} \in X$ in $\bar{U}\left(x_{0}, r\right)$ if $F$ is twice Fréchet-differentiable, and $F^{\prime}\left(x_{0}\right)^{-1}$ exists such that

$$
\left\|F^{\prime}\left(x_{0}\right)^{-1} F^{\prime \prime}(x)\right\| \leq \frac{2 \gamma}{\left(1-\gamma\left\|x-x_{0}\right\|\right)^{3}} \text { for all } x \in \bar{U}\left(x_{0}, r\right) .
$$

Wang showed that the above $\gamma$-condition is weaker than the criterion point estimate

$$
\gamma\left(F, x_{0}\right) \leq \gamma,
$$

where $\gamma\left(F, x_{0}\right)$ is given by $(1.5)$. Moreover he showed that the conclusions of Theorem 1.2 hold with $\gamma$ replacing $\gamma\left(F, x_{0}\right)$.

Here using more precise majorizing sequences and the same or even weaker hypotheses we provide a semilocal convergence analysis with the following advantages:

(a) finer error estimates on the distances $\left\|x_{n+1}-x_{n}\right\|,\left\|x_{n}-x^{*}\right\|(n \geq 0)$ and

(b) at least as precise information on the location of the solution $x^{*}$.

Finally we study the local convergence of Newton's method not examined in [10].

\section{Semilocal Convergence Analysis of Newton's Method}

We introduce the $\left(\gamma_{0}, \gamma\right)$ condition:

Definition 2.1. Suppose:

$$
0<\gamma_{0} \leq \gamma
$$

We say $F$ satisfies the $\left(\gamma_{0}, \gamma\right)$ condition at $x_{0} \in D$ in $\bar{U}\left(x_{0}, r\right) \subseteq D$ if operator $F$ is Fréchet-differentiable, $F^{\prime}\left(x_{0}\right)^{-1}$ exists such that for $r<\frac{1}{\gamma_{0}}$

$$
\left\|F^{\prime}\left(x_{0}\right)^{-1}\left[F^{\prime}\left(x_{t}\right)-F^{\prime}(y)\right]\right\| \leq \frac{2 \gamma\left\|x_{t}-y\right\|}{\left(1-\gamma\left\|x_{t}-x_{0}\right\|\right)^{3}},
$$


and

$$
\left\|F^{\prime}\left(x_{0}\right)^{-1}\left[F^{\prime}(x)-F^{\prime}\left(x_{0}\right)\right]\right\| \leq \frac{1}{\left(1-\gamma_{0}\left\|x-x_{0}\right\|\right)^{2}}-1
$$

for all $x, y \in \bar{U}\left(x_{0}, r\right), t \in[0,1]$ and $x_{t}=y+t(x-y)$.

Remark 2.2. Note that even if $\gamma_{0}=\gamma$ and $F$ is a twice Fréchet-differentiable operator (2.2) in Definition 2.1 is still weaker than (1.17) in Definition 1.3. In view of $(2.2)$ it follows that there exists $\gamma_{1} \in(0, \gamma]$ such that

$$
\left\|F^{\prime}\left(x_{0}\right)^{-1}\left[F^{\prime}\left(x_{t}\right)-F^{\prime}\left(x_{0}\right)\right]\right\| \leq \frac{2 \gamma_{1}\left\|x_{t}-x_{0}\right\|}{\left(1-\gamma_{1}\left\|x_{t}-x_{0}\right\|\right)^{3}}
$$

It is convenient for us to introduce scalar sequences $\left\{t_{n}\right\},\left\{r_{n}\right\}$ for $t_{0}=$ $r_{0}=0, t_{1}=\beta$ by

$$
t_{n+1}=t_{n}-\frac{\left(1-\gamma_{0} t_{n}\right)^{2} g\left(t_{n-1}, t_{n}\right)}{1-2\left(1-\gamma_{0} t_{n}\right)^{2}} \quad(n \geq 1),
$$

and

$$
r_{n+1}=r_{n}-\frac{\left(1-\gamma_{0} r_{n}\right)^{2} f\left(r_{n}\right)}{1-2\left(1-\gamma_{0} r_{n}\right)^{2}} \quad(n \geq 0)
$$

where,

$$
g(v, w)=\frac{\gamma[2-\gamma(v+w)](w-v)^{2}}{[(1-\gamma v)(1-\gamma w)]^{2}}, \text { for } \gamma \neq v, \gamma \neq w
$$

Then using the definitions of sequences $\left\{s_{n}\right\},\left\{t_{n}\right\},\left\{r_{n}\right\}$ and induction on $n \geq 0$, it can easily be seen that the following result for majorizing sequences holds:

Lemma 2.3. If $\gamma_{0}<\gamma$, and

$$
\alpha=\beta \gamma \leq 3-2 \sqrt{2},
$$

then for all $n \geq 1$ the following estimates hold:

$$
\begin{aligned}
0 \leq t_{n} & <r_{n}<s_{n}, \\
0<t_{n+1}-t_{n} & <r_{n+1}-r_{n}<s_{n+1}-s_{n}, \\
0 \leq t^{*}-t_{n} & \leq r^{*}-r_{n} \leq s^{*}-s_{n},
\end{aligned}
$$

and

$$
t^{*} \leq r^{*} \leq s^{*}
$$


Remark 2.4. Clearly if $\gamma_{0}=\gamma$, then

$$
t_{n}=r_{n}=s_{n} \quad(n \geq 0)
$$

holds.

We can show the main semilocal convergence theorem for Newton's method (1.2):

Theorem 2.5. Under the hypotheses of Lemma 2.3 if $F$ satisfies the $\left(\gamma_{0}, \gamma\right)$ condition at $x_{0} \in D$ in $\bar{U}\left(x_{0}, s^{*}\right) \subseteq D$ then, sequence $\left\{x_{n}\right\}(n \geq 0)$ generated by Newton's method (1.2) is well defined, remains in $\bar{U}\left(x_{0}, t^{*}\right)$ for all $n \geq 0$ and converges to a unique solution $x^{*} \in \bar{U}\left(x_{0}, t^{*}\right)$ of equation $F(x)=0$.

Moreover the following estimates hold true for all $n \geq 0$

$$
\left\|x_{n+1}-x_{n}\right\| \leq t_{n+1}-t_{n}
$$

and

$$
\left\|x_{n}-x^{*}\right\| \leq t^{*}-t_{n}
$$

Furthermore, if there exists $R>s^{*}$ such that $U\left(x_{0}, R\right) \subseteq D$, where

$$
R=\frac{1}{\gamma_{1}}\left[1-\frac{1}{2\left(1-\gamma_{1} s^{*}\right)}\right]
$$

then the solution $x^{*}$ is unique in $U\left(x_{0}, R\right)$.

Proof. We shall show

$$
\left\|x_{k+1}-x_{k}\right\| \leq t_{k+1}-t_{k}
$$

and

$$
\bar{U}\left(x_{k+1}, t^{*}-t_{k+1}\right) \subseteq \bar{U}\left(x_{k}, t^{*}-t_{k}\right)
$$

hold for all $k \geq 0$.

For every $z \in \bar{U}\left(x_{1}, t^{*}-t_{1}\right)$,

$$
\left\|z-x_{0}\right\| \leq\left\|z-x_{1}\right\|+\left\|x_{1}-x_{0}\right\| \leq t^{*}-t_{1}+t_{1}=t^{*}-t_{0}
$$

implies $z \in \bar{U}\left(x_{0}, t^{*}-t_{0}\right)$. We also have

$$
\left\|x_{1}-x_{0}\right\|=\left\|F^{\prime}\left(x_{0}\right)^{-1} F\left(x_{0}\right)\right\|=\beta=t_{1}-t_{0} .
$$

Therefore (2.16) and (2.17) hold for $k=0$. 
Given (2.16) and (2.17) hold for $n=0,1, \ldots, k-1$, then

$$
\left\|x_{k}-x_{0}\right\| \leq \sum_{i=1}^{k}\left\|x_{i}-x_{i-1}\right\| \leq \sum_{i=1}^{k}\left(t_{i}-t_{i-1}\right)=t_{k}-t_{0}=t_{k}
$$

and

$$
\begin{gathered}
\left\|x_{k-1}+t\left(x_{k}-x_{k-1}\right)-x_{0}\right\| \leq t_{k-1}+t\left(t_{k}-t_{k-1}\right),<t^{*} \\
t \in[0,1] .
\end{gathered}
$$

In view of (1.2) we obtain the approximation

$$
F\left(x_{k}\right)=F\left(x_{k}\right)-F\left(x_{k-1}\right)-F^{\prime}\left(x_{k-1}\right)\left(x_{k}-x_{k-1}\right) .
$$

By (1.12), (2.2), (2.4)-(2.7), (2.9), (2.16) and (2.18) we can have in turn:

$$
\begin{aligned}
\left\|F^{\prime}\left(x_{0}\right)^{-1} F\left(x_{k}\right)\right\|= & \| F^{\prime}\left(x_{0}\right)^{-1} \int_{0}^{1}\left[F^{\prime}\left(x_{k-1}+t\left(x_{k}-x_{k-1}\right)\right)\right. \\
& \left.-F^{\prime}\left(x_{k-1}\right)\right]\left(x_{k}-x_{k-1}\right) \| \\
\leq & 2 \gamma \int_{0}^{1} \frac{t\left\|x_{k}-x_{k-1}\right\| d t}{\left[1-\gamma\left\|x_{k-1}-x_{0}+t\left(x_{k}-x_{k-1}\right)\right\|\right]^{3}} \\
\leq & 2 \gamma \int_{0}^{1} \frac{t\left(t_{k}-t_{k-1}\right) d t}{\left[1-\gamma\left(t_{k-1}+t\left(t_{k}-t_{k-1}\right)\right]^{3}\right.} \\
= & g\left(t_{k-1}, t_{k}\right) .
\end{aligned}
$$

It also follows from (2.3), (2.7), (2.16)-(2.18) that

$$
\begin{aligned}
\| F^{\prime}\left(x_{0}\right)^{-1} & {\left[F^{\prime}\left(x_{k}\right)-F^{\prime}\left(x_{0}\right)\right] \| } \\
& \leq \frac{1}{\left(1-\gamma_{0}\left\|x_{k}-x_{0}\right\|\right)^{2}}-1 \leq \frac{1}{\left(1-\gamma_{0} t_{k}\right)^{2}}-1 \\
& <\frac{1}{\left(1-\gamma_{0} t^{*}\right)^{2}}-1 \leq 1 .
\end{aligned}
$$

It follows from (2.22) and the Banach Lemma on invertible operators [3], [7] that $F^{\prime}\left(x_{k}\right)^{-1}$ exists and

$$
\begin{aligned}
\left\|F^{\prime}\left(x_{k}\right)^{-1} F^{\prime}\left(x_{0}\right)\right\| & \leq\left[1-\left(\frac{1}{\left(1-\gamma_{0}\left\|x_{k}-x_{0}\right\|\right)^{2}}-1\right)\right]^{-1} \\
& \leq\left[1-\left(\frac{1}{\left(1-\gamma_{0} t_{k}\right)^{2}}-1\right)\right]^{-1}
\end{aligned}
$$


It follows by (1.2), (2.21) and (2.23) that

$$
\begin{aligned}
\left\|x_{k+1}-x_{k}\right\| & =\left\|F^{\prime}\left(x_{k}\right)^{-1} F\left(x_{k}\right)\right\| \leq\left\|F^{\prime}\left(x_{k}\right)^{-1} F^{\prime}\left(x_{0}\right)\right\| \cdot\left\|F^{\prime}\left(x_{0}\right)^{-1} F\left(x_{k}\right)\right\| \\
(2.24) & \leq \frac{\left(1-\gamma_{0} t_{k}\right)^{2}}{g\left(t_{k-1}, t_{k}\right) \cdot\left[-1+2\left(1-\gamma_{0} t_{k}\right)^{2}\right]}=t_{k+1}-t_{k} .
\end{aligned}
$$

Thus for every $z \in \bar{U}\left(x_{k+1}, t^{*}-t_{k+1}\right)$ we have

$$
\left\|z-x_{k}\right\| \leq\left\|z-x_{k+1}\right\|+\left\|x_{k+1}-x_{k}\right\| \leq t^{*}-t_{k+1}+t_{k+1}-t_{k}=t^{*}-t_{k} .
$$

That is,

$$
z \in \bar{U}\left(x_{k}, t^{*}-t_{k}\right) .
$$

Estimates (2.24) and (2.25) imply that (2.16) and (2.17) hold for $n=k$. Therefore the induction for (2.16) and (2.17) is completed.

Lemma 2.3 implies that $\left\{t_{n}\right\}(n \geq 0)$ is a Cauchy sequence. In view of (2.16) and (2.17) $\left\{x_{n}\right\}$ becomes a Cauchy sequence too, and as such it converges to some $x^{*} \in \bar{U}\left(x_{0}, t^{*}\right)$ (since $\bar{U}\left(x_{0}, t^{*}\right)$ is a closed set). Estimate (2.14) follows from (2.13) by using standard majorization techniques [3], [7]. By letting $k \rightarrow \infty$ in $(2.21)$ we obtain $F\left(x^{*}\right)=0$.

To show uniqueness in $\bar{U}\left(x_{0}, t^{*}\right)$, let $y^{*} \in \bar{U}\left(x_{0}, t^{*}\right)$ be a solution of equation $F(x)=0$. We shall show for all $k \geq 0$

$$
\left\|x_{k}-y^{*}\right\| \leq t^{*}-t_{k} .
$$

For $k=0(2.26)$ holds true by the initial conditions. Let us assume that (2.26) holds true for $n=0,1, \ldots, k-1$.

As in (2.21) and (2.22) with $x_{k}, y^{*}$ replacing $x_{k-1}, x_{k}$ respectively and using the induction hypothesis and the approximation

$$
\begin{aligned}
x_{k+1}-y^{*} & =x_{k}-F^{\prime}\left(x_{k}\right)^{-1} F\left(x_{k}\right)-y^{*} \\
& =F^{\prime}\left(x_{k}\right)^{-1}\left[F\left(x^{*}\right)-F\left(x_{k}\right)-F^{\prime}\left(x_{k}\right)\left(y^{*}-x_{k}\right)\right] \\
& =F^{\prime}\left(x_{k}\right)^{-1} \int_{0}^{1}\left[F^{\prime}\left(x_{k}+t\left(y^{*}-x_{k}\right)\right)-F^{\prime}\left(x_{k}\right)\right]\left(y^{*}-x_{k}\right) d t
\end{aligned}
$$

we obtain in turn

$$
\begin{aligned}
\left\|x_{k+1}-y^{*}\right\| \leq & \left\|F^{\prime}\left(x_{k}\right)^{-1} F^{\prime}\left(x_{0}\right)\right\| \\
& \cdot\left\|F^{\prime}\left(x_{0}\right)^{-1} \int_{0}^{1}\left[F^{\prime}\left(x_{k}+t\left(y^{*}-x_{k}\right)\right)-F^{\prime}\left(x_{k}\right)\right]\right\|\left\|y^{*}-x_{k}\right\| d t \\
\leq & \frac{\left(1-\gamma_{0}\left\|x_{k}-x_{0}\right\|\right)^{2}}{2 \gamma\left[-1+2\left(1-\gamma_{0}\left\|x_{k}-x_{0}\right\|\right)^{2}\right]} \int_{0}^{1} \frac{t\left\|x_{k}-y^{*}\right\| d t}{\left[1-\gamma\left(\left\|x_{k}+t\left(y^{*}-x_{k}\right)\right\|\right)\right]^{3}}
\end{aligned}
$$




$$
\begin{aligned}
& \leq \frac{\left(1-\gamma_{0} t_{k}\right)^{2}}{2 \gamma\left[-1+2\left(1-\gamma_{0} t_{k}\right)^{2}\right]} \int_{0}^{1} \frac{t\left(t^{*}-t_{k}\right) d t}{\left[1-\gamma\left(t_{k}+t\left(t^{*}-t_{k}\right)\right)\right]^{3}} \\
& \leq t^{*}-t_{k+1}
\end{aligned}
$$

which shows (2.26) for all $k \geq 0$. By letting $k \rightarrow \infty$ in (2.26) we obtain $\lim _{k \rightarrow \infty} x_{k}=y^{*}$. However we know $\lim _{k \rightarrow \infty} x_{k}=x^{*}$. Hence, we conclude $x^{*}=y^{*}$.

Finally to show uniqueness in $U\left(x_{0}, R\right)$, let $y^{*}$ be a solution of equation $F(x)=0$ in $U\left(x_{0}, R\right)$. Define linear operator $L$ by

$$
L=\int_{0}^{1} F^{\prime}\left(x^{*}+t\left(y^{*}-x^{*}\right)\right) d t .
$$

Using $(2.2)^{\prime}$ and (2.15) we obtain in turn

$$
\begin{aligned}
\left\|F^{\prime}\left(x_{0}\right)^{-1}\left[F^{\prime}\left(x_{0}\right)-L\right]\right\| \\
\quad=\left\|F^{\prime}\left(x_{0}\right)^{-1} \int_{0}^{1}\left[F^{\prime}\left(x^{*}+t\left(y^{*}-x^{*}\right)\right)-F^{\prime}\left(x_{0}\right)\right] d t\right\| \\
\quad \leq \int_{0}^{1} \frac{2 \gamma_{1}\left\|x^{*}+t\left(y^{*}-x^{*}\right)-x_{0}\right\| d t}{\left[1-\gamma_{1}\left\|x^{*}+t\left(y^{*}-x^{*}\right)-x_{0}\right\|\right]^{3}} \\
\quad \leq \int_{0}^{1} \frac{2 \gamma_{1}\left[\left\|x^{*}-x_{0}\right\| t+(1-t)\left\|y^{*}-x_{0}\right\| d t\right.}{\left[1-\gamma_{1}\left(\left\|x^{*}-x_{0}\right\| t+(1-t)\left\|y^{*}-x_{0}\right\|\right]^{3}\right.} \\
\quad<\int_{0}^{1} \frac{2 \gamma_{1}[t r+(1-t) R] d t}{\left.1-\gamma_{1}(t r+(1-t) R)\right]^{3}} \\
\quad \leq \frac{1}{(1-\gamma r)(1-\gamma R)}-1=1
\end{aligned}
$$

by the choice of $R$. In view of (2.30) and the Banach Lemma on invertible operators we deduce operator $L$ is invertible.

Finally using the identity

$$
F\left(y^{*}\right)-F\left(x^{*}\right)=L\left(y^{*}-x^{*}\right)
$$

we conclude that $x^{*}=y^{*}$.

That completes the proof of the theorem.

Remark 2.6. Under the hypotheses of Theorem 2.5, we showed in Lemma 2.3 that $\left\{t_{n}\right\}$ is a finer majorizing sequence than $\left\{s_{n}\right\}$ used in [10]. One expects that sequence $\left\{t_{n}\right\}$ converges under hypotheses weaker than (2.7). In [2] (see also [1]) we provided sufficient convergence conditions for more general sequences than $\left\{t_{n}\right\}$. That is why we refer the reader there and we do 
not pursue this problem further but instead we study the local convergence of Newton's method (1.2).

We now complete this section with a simple numerical example.

Example 2.7. Let $X=Y=\mathbf{R}, \beta=1, \gamma_{0}=\gamma=\alpha>0, D=\left[0, \frac{1}{\gamma}\right)$, and define function $f$ on $D$ by

$$
f(t)=1-t+\frac{\gamma t^{2}}{1-\gamma t} .
$$

Let $\alpha=\frac{1}{2}(3-2 \sqrt{2})=.0857864$, then we get

$$
t^{*}=s^{*}=r^{*}=1.119 .
$$

\section{Local Convergence of Newton's Method}

In this section we assume: there exist a solution $x^{*}$ of equation $F(x)=0$ and positive constants $\delta_{0}<\delta$ such that for all $t \in[0,1], x \in D$ the following hold true:

$$
F^{\prime}\left(x^{*}\right)^{-1} \in L(Y, X)
$$

$$
\begin{aligned}
\left\|F^{\prime}\left(x^{*}\right)^{-1}\left[F^{\prime}(x)-F^{\prime}\left(x^{*}\right)\right]\right\| & \leq \frac{1}{\left(1-\delta_{0}\left\|x-x^{*}\right\|\right)^{2}}-1, \\
\left\|F^{\prime}\left(x^{*}\right)^{-1}\left[F^{\prime}\left(y_{t}\right)-F^{\prime}(x)\right]\right\| & \leq \frac{2 \delta\left\|y_{t}-x\right\|}{\left(1-\delta\left\|y_{t}-x^{*}\right\|\right)^{3}}, \\
U\left(x^{*}, \frac{1}{\delta_{0}}\right) & \subseteq D
\end{aligned}
$$

where, $y_{t}=x+t\left(x^{*}-x\right)$.

Clearly, there exists $a \geq 1$ such that $\delta=a \delta_{0}$. Define scalar function $h$ on $[0,1]$ for each fixed $a$ by

$$
h(s)=a^{2} s^{4}-2 a(a+3) s^{3}+2(6 a+1) s^{2}-4(1+a) s+1 .
$$

It follows by the intermediate value theorem, the first derivative test that since $h(0) h(1)=-(a-1)^{2} \leq 0$ and $h^{\prime}(s) \leq 0$ on [0,1], function $h$ has a unique zero $s^{*}=s^{*}(a)$ in $(0,1)$ for all $a \geq 1$, and

$$
h(s) \geq 0 \text { for all } s \in\left[0, s^{*}\right] .
$$

Note that in particular if $a=1$, then

$$
s^{*}=s^{*}(1)=3-2 \sqrt{2},
$$


since, $h$ becomes

$$
h(s)=(1-s)^{2}[s-(3-2 \sqrt{2})][s-(3+2 \sqrt{2})] .
$$

We can show the following local convergence result for Newton's method (1.2):

Theorem 3.1. Under hypotheses (3.1)-(3.4), sequence $\left\{x_{n}\right\}$ generated by Newton's method (1.2) starting at $x_{0} \in U\left(x^{*}, R\right)$ is well defined, remains in $U\left(x^{*}, R\right)$ for all $n \geq 0$ and converges to $x^{*}$, where

$$
R=\frac{s^{*}}{\delta_{0}}
$$

Moreover the following errob bounds hold for all $n \geq 0$ :

$$
\left\|x_{n+1}-x^{*}\right\| \leq p\left(\delta_{0}, \delta, R\right)\left\|x_{n}-x^{*}\right\|^{2},
$$

where,

$$
p\left(\delta_{0}, \delta, R\right)=\frac{(2+\delta R)\left(1-\delta_{0} R\right)^{2} \delta}{\left[2\left(1-\delta_{0} R\right)^{2}-1\right](1-\delta R)}
$$

Proof. We first show that $F^{\prime}(x)^{-1} \in L(Y, X)$ for all $x \in \bar{U}\left(x^{*}, R\right)$. In view of (3.2) we get

$$
\begin{gathered}
\| F^{\prime}\left(x^{*}\right)^{-1}\left[F^{\prime}(x)-F^{\prime}\left(x^{*}\right)\right] \leq \frac{1}{\left(1-\delta_{0}\left\|x-x^{*}\right\|\right)^{2}}-1 \\
\leq \frac{1}{\left(1-\delta_{0} R\right)^{2}}-1<1
\end{gathered}
$$

by the choice of $R$.

It follows from (3.12) and the Banach Lemma on invertible operators that

$$
\begin{aligned}
\left\|F^{\prime}(x)^{-1} F^{\prime}\left(x^{*}\right)\right\| & \leq \frac{1}{1-\left[\frac{1}{\left(1-\delta_{0}\left\|x-x^{*}\right\|\right)^{2}}-1\right]} \\
& \leq \frac{1}{1-\left[\frac{1}{\left(1-\delta_{0} R\right)^{2}}-1\right]}=\frac{\left(1-\delta_{0} R\right)^{2}}{2\left(1-\delta_{0} R\right)^{2}-1}
\end{aligned}
$$


We shall show $x_{n} \in U\left(x^{*}, R\right)$ for all $n \geq 0$. In view of the initial condition $x_{0} \in U\left(x^{*}, R\right)$. Let us assume $x_{k} \in U\left(x^{*}, R\right), k=0,1, \ldots, n$. Using (1.2) we obtain the approximation

$$
\begin{aligned}
x_{k+1}-x^{*}= & x_{k}-F^{\prime}\left(x_{k}\right)^{-1} F\left(x_{k}\right)-x^{*} \\
= & F^{\prime}\left(x_{k}\right)^{-1}\left[F\left(x^{*}\right)-F\left(x_{k}\right)-F^{\prime}\left(x_{k}\right)\left(x^{*}-x_{k}\right)\right] \\
= & -\left[F^{\prime}\left(x_{k}\right)^{-1} F^{\prime}\left(x^{*}\right)\right] \int_{0}^{1}\left[F^{\prime}\left(x_{k}\right) t\left(x^{*}-x_{k}\right)\right) \\
& \left.-F^{\prime}\left(x_{k}\right)\right]\left(x^{*}-x_{k}\right) d t .
\end{aligned}
$$

By the induction hypotheses, (3.3), (3.13) and (3.14) we obtain in turn

$$
\begin{aligned}
\left\|x_{k+1}-x^{*}\right\| & \leq \frac{\left(1-\delta_{0} R\right)^{2}}{\left[2\left(1-\delta_{0} R\right)^{2}-1\right]} \int_{0}^{1} \frac{2 \delta t\left\|x_{k}-x^{*}\right\|^{2} d t}{\left[1-\delta(1-t)\left\|x_{k}-x^{*}\right\|\right]^{3}} \\
& \leq \frac{\left(1-\delta_{0} R\right)^{2}}{\left[2\left(1-\delta_{0} R\right)^{2}-1\right]}\left[-1+\frac{1}{\left(1-\delta\left\|x_{k}-x^{*}\right\|\right)^{2}}\right]\left\|x_{k}-x^{*}\right\| \\
& <\frac{\left(1-\delta_{0} R\right)^{2}}{\left[2\left(1-\delta_{0} R\right)^{2}-1\right]}\left[-1+\frac{1}{(1-\delta R)^{2}}\right]\left\|x_{k}-x^{*}\right\|^{2} \\
& \leq p\left(\delta_{0}, \delta, R\right)\left\|x_{k}-x^{*}\right\|^{2} \\
& <p\left(\delta_{0}, \delta, R\right) R\left\|x_{k}-x^{*}\right\|<\left\|x_{k}-x^{*}\right\|,
\end{aligned}
$$

since,

$$
p\left(\delta_{0}, \delta, R\right) R \leq 1
$$

by (3.6) for $s=\delta_{0} R$.

Finally, from (3.15) it follows that (3.10) holds for all $n \geq 0, \lim _{k \rightarrow \infty} x_{k}=$ $x^{*}$ and $x_{k+1} \in U\left(x^{*}, R\right)$.

That completes the proof of Theorem 3.1.

Remark 3.2. In the special case when $\delta_{0}=\delta=\gamma\left(F, x^{*}\right), a=1[9]$ the radius of convergence $R_{0}$ was found to be

$$
R_{0}=\frac{1}{2 \delta}(3-2 \sqrt{2}) \leq \frac{R}{2} .
$$

Therefore we have doubled the radius of convergence for Newton's method under the same computational cost, and under weaker hypotheses than before [9]. 


\section{References}

[1] Argyros, I. K., A convergence analysis for Newton's method based on Lipschitz center-Lipschitz and analytic operators, Pan American Math. J. 13, 3, pp. 19-24, (2003).

[2] Argyros, I. K., A unifying local-semilocal convergence analysis and applications for two-point Newton-like methods in Banach space, $J$. Math. Anal. Applic. 298, pp. 374-397, (2004).

[3] Argyros, I. K., Approximate Solution of Operator Equations with Applications, World Scientific Publ. Comp., Hackensack,, New Jersey, U.S.A., (2005)

[4] Dedieu, J. P. and Shub, M., Multihomogeneous Newton methods, Math. Comput. 69, 231, pp. 1071-1098, (1999).

[5] Ezquerro, J. A. and Hernandez, M.A., On a convex acceleration of Newton's method, J. Optim. Th. Appl. 100, 2, pp. 311-326, (1999).

[6] Gutierrez, J. M., A new semilocal convergence theorem for Newton's method, J. Comput. Appl. Math. 79, pp. 131-145, (1997).

[7] Kantorovich, L. V. and Akilov, G.P., Functional Analysis in Normed Spaces, Pergamon Press, Oxford, (1982).

[8] Smale, S., Newton's method estimate from data at one point, in The Merging of Disciplines: New Directions in Pure, Applied and Computational Mathematics (eds., Ewing, R. et al.), Springer-Verlag, New York, (1986).

[9] Wang, D. and Zhao, F., The theory of Smale's point estimation and its applications, J. Comput. Appl. Math. 60, pp. 253-269, (1995).

[10] Wang, X. H. and Han, D.F., On dominating sequence method in the point estimate and Smale theorem, Sci. Sinica Ser. A, 33, pp 135144, (1990).

[11] Wang, X. H., Convergence of the iteration of Halley family in weak conditions, Chinese Science Bulletin, 42, pp. 552-555, (1997). 
Ioannis K. Argyros

Department of Mathematical Sciences

Cameron University

Lawton, OK 73505

U. S. A.

e-mail address : iargyros@cameron.edu 